\title{
Ball under action of periodic point load
}

Alexeev Gennady Valentinovich

Department of Process and Equipment in Food Production St. Petersburg National Research University of Information Technology, Mechanics and Optics

St. Petersburg, Russia gva2003@mail.ru

\section{Voronenko Borukh Evseevich}

Department of Process and Equipment in Food Production St. Petersburg National Research University of Information Technology, Mechanics and Optics

St. Petersburg, Russia

\author{
Verboloz Elena Igorevna \\ Department of Process and Equipment in Food Production \\ St. Petersburg National Research University of Information \\ Technology, Mechanics and Optics \\ St. Petersburg, Russia \\ elenaverboloz@mail.ru
}

\section{Loza Alexander Alexandrovich}

Department of Process and Equipment in Food Production

St. Petersburg National Research University of Information Technology, Mechanics and Optics St. Petersburg, Russia projecttechnic@mail.ru

\begin{abstract}
Technological equipment in various industries uses an abrasive effect on the object being processed, and the continuity of the contact of the working organs with it is often disrupted. Sometimes such regime is maintained intentionally, that is, periodic contact of the treated object with the abrasive is ensured. In such cases, due to the prevention of overheating, the processed raw materials are sometimes more fully used, for example, in the food industry; the preservation of its nutritional value (vitamins, native gluten properties, etc.) is ensured. To solve the problem of observing a certain temperature regime, it is necessary to carry out some analytical estimates [1-5].
\end{abstract}

Keywords - technological equipment; working organs; contact of the treated object with an abrasive; prevention of overheating; preservation of its nutritional value.

\section{INTRODUCTION}

Consideration of movement trajectories of the processed food product in the specific working chambers, indicating that it may touch the abrasive tool at various points. Thus, thermal contact with the working organs is carried out only periodically and accidentally as well as with heated related objects.

The described physical model can be formalized in the form of a thermal problem of heat transfer in a resting ball subjected to periodic point contacts with a heat source [6].

Let us write the statement of the classical heat problem for the physical model considered, assuming that the contact is carried out in the infinite number of points in the form of:

$$
u_{\mathrm{t}=} \alpha_{2} u_{\mathrm{xx}, 0} \leq x \leq \infty, 0 \leq t \leq \infty
$$

initial condition: $u_{(\mathrm{x}, 0)}=u_{\mathrm{o}, 0} \leq x \leq \infty$

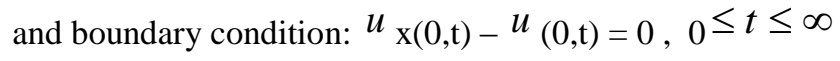

The boundary value problem will be solved by using the Laplace transformation through variable $t$ [7].

At the first stage of the solution, let us find the images of the partial derivatives entering equation (1)

$$
\begin{gathered}
\mathrm{L}\left[u_{\mathrm{t}}\right]=\int_{0}^{\infty} u_{t}(x, t) e^{-s t} d t=\left.u(x, t) e^{-s t}\right|_{0} ^{\infty}+ \\
s \int_{0}^{\infty} u(x, t) e^{-s t} d t=s U(x, s)-u(x, 0), \\
\mathrm{L}\left[u_{\mathrm{x}}\right]=\int_{0}^{\infty} u_{X} e^{-s t} d t=\frac{\partial}{\partial x} U(x, s),
\end{gathered}
$$




$$
\mathrm{L}\left[u_{X X}\right]=\int_{0}^{\infty} u_{X X} e^{-s t} d t=\frac{\partial^{2}}{\partial x^{2}} U(x, s),
$$

Let us substitute the expressions for the partial derivatives in equation (1) for the transition to ordinary differential equation [8]:

$$
s U(x)-u_{0}=\frac{d^{2} U}{d x^{2}}, 0 \leq x \leq \infty
$$

The initial data for equation (4) can be found by transforming (2):

$$
\frac{d U}{d x}(0)=U(0)
$$

Equation (4) is an ordinary differential equation of the second order; therefore, in order to solve the corresponding boundary-value problem for a given equation, it is necessary to specify another boundary condition, which is sufficiently obvious from physical considerations:

$$
U(x) \rightarrow 0 \text { with } x \rightarrow+\infty
$$

The general solution of (4) is obtained by summing the general solution of the homogeneous equation [9]:

$$
\frac{d^{2} U}{d x^{2}}-s U(x)=0
$$

and the particular solution of the inhomogeneous equation: $u_{0}$ :

$$
U(x)=c_{1} e^{\sqrt{s x}}+c_{2} e^{-\sqrt{s x}}+\frac{u_{0}}{s}
$$

From physical considerations, let us find constant $c_{1}$, and from the boundary conditions for equation (4), it follows that constant $c_{2}$ is a solution of equation

$$
-c_{2} \sqrt{s}=c_{2}+\frac{u_{0}}{s}
$$

obtained by substituting (5) into (4):

$$
c_{2}=\frac{u_{0}}{s(\sqrt{s}+1} \text {. }
$$

Finally, substituting the obtained values of both constants c, there is the expression for $U(x)$ in the form of:

$$
U(x)=u_{0}\left(1-\frac{1}{s(\sqrt{s}+1)}\right)
$$

The temperature field $u(x, t)$ is determined by calculating the following integral [10]:

$$
\begin{gathered}
u(x, t)=L^{-1}[U(x, s)]= \\
\int_{c-i \infty}^{c+i \infty} U(s) e^{s t} d s=\int_{c-i \infty}^{c+i \infty}\left(\frac{1}{s}-\frac{1}{s(\sqrt{s}+1)}\right) e^{s t} d s
\end{gathered}
$$

For example, the methods of complex variable functions can be used.

The result of calculating this integral is written as:

$$
\begin{gathered}
u(x, t)=u_{0}-u_{0}\left[\operatorname{erfc}\left(\frac{x}{2 \sqrt{t}}\right)+\operatorname{erfc}\left(\sqrt{t}+\frac{x}{2 \sqrt{t}}\right) e^{x+t}\right] \\
\operatorname{erfc}(x)=\frac{2}{\sqrt{\pi}} \int_{x}^{\infty} e-\xi^{2} d \xi \\
\text { where }
\end{gathered}
$$

is a function which is complementary to the probability integral. Thus, relation (9) is a solution of boundary value problem (1), assuming an infinite number of contacts throughout a semi-infinite given domain of definition of the variable parameters.

\section{CARRIED OUT RESEARCH AND DISCUSSION OF RESULTS}

If, as a refined model of a heated object, let us use a sphere to which thermal impulses are periodically fed from friction against abrasive sections of the working organs of the process equipment, the thermal process that occurs during the exfoliation of cereals can be described by the known heat equation for a spherical body [11]:

$$
\left.\frac{\partial t}{\partial \tau}=a\left(\frac{\partial^{2} t}{\partial r^{2}}+\frac{2}{r} \frac{\partial t}{\partial r}\right)_{,(0<} r<R, \tau>0\right)
$$


where $\mathrm{t}(r, \tau)$ - the temperature;

$\tau$ - the time;

$r$ - the radius of the processed product;

$\mathrm{R}$ - the radius of the working chamber of the machine or workpiece;

$a$ - the coefficient of thermal diffusivity;

The initial temperature of the process is constant:

$$
\mathrm{t}(\mathrm{r}, \mathrm{o})=\text { to }=\text { const }
$$

The boundary conditions, under which the process proceeds, are boundary conditions of the first kind [12]:

$$
t(R, \tau)= \begin{cases}t_{1}=t_{\min } & \text { для } 0<\tau<\tau_{1} ; \\ t_{2}=t_{m p}=t_{\max } & \text { для } \tau_{1}<\tau<\tau_{2} \quad\left(t_{1}>t_{2}\right) ;\end{cases}
$$

In view of the symmetry condition of the object, let us write down the boundary condition of the second kind in the form of:

$$
\frac{\partial t(0, \tau)}{\partial r}=0
$$

And in addition, the condition of the limited temperature in the center of the heated object is:

$$
t(0, \tau)<\infty
$$

Period $\mathrm{T}=\tau 2(0<\tau 2<\mathrm{T}), \Delta \tau=\tau 2-\tau 1$.

The number of cycles is:

$$
\mathrm{N}=\frac{2 \pi R}{L}
$$

where $L$ is the length of the part of the outer circumference of the object that periodic contacts of the abrasive cover during period $\mathrm{T}$. The boundary-value problem of this kind was solved by the Laplace integral transformation method, and the distribution of the temperature fields in the object was obtained in the following dimensionless form:

$$
\begin{gathered}
T(X, F O)=1-\frac{F_{O_{1}}}{F_{O_{2}}}+\sum_{n=1}^{\infty}(-1)^{n} \frac{2\left(1-e^{-(n \pi)^{2} F_{2} O_{2}^{\left(1-\frac{F_{0_{1}}}{F_{0_{2}}}\right.}}\right)}{1-e^{-(n \pi)^{2} F_{0_{2}}}} \cdot \frac{\sin (n \pi X)}{\pi n X} \cdot e^{-(n \pi)^{2} F_{0_{1}}-} \\
-\sum_{m=1}^{\infty} \frac{\left(P_{1} \cos \left(\frac{2 \pi m}{F_{0_{2}}}\left(F O-\frac{F_{0_{1}}}{2}\right)\right)+P_{2} \sin \left(\frac{2 \pi m}{F_{0_{2}}}\left(F_{O}-\frac{F_{0_{1}}}{2}\right)\right)\right)}{\operatorname{ch}\left(2 \sqrt{\frac{\pi m}{F O_{2}}}\right)-\cos \left(2 \sqrt{\frac{\pi m}{F o_{2}}}\right)} \cdot \frac{\sin \left(\pi m \frac{F_{0_{1}}}{F_{0_{2}}}\right.}{\pi m X},(16) \\
\text { where }
\end{gathered}
$$
temperature, $0<\mathrm{T}<1$;

$$
\begin{gathered}
P_{1}=\operatorname{sh} \sqrt{\frac{\pi m}{F_{0_{2}}}} \cos \sqrt{\frac{\pi m}{F_{0_{2}}}} \operatorname{sh}\left(\sqrt{\frac{\pi m}{F_{0_{2}}}} X\right) \cos \left(\sqrt{\frac{\pi m}{F_{0_{2}}}} X\right)+\operatorname{ch} \sqrt{\frac{\pi m}{F_{0_{2}}}} \sin \sqrt{\frac{\pi m}{F_{0_{2}}}} \operatorname{ch}\left(\sqrt{\frac{\pi m}{F_{0}}} X\right) \sin \left(\sqrt{\frac{\pi m}{F_{0_{2}}}} X\right) ; \\
P_{2}=\operatorname{ch} \sqrt{\frac{\pi m}{F_{0_{2}}}} \sin \sqrt{\frac{\pi m}{F_{0_{2}}}} \operatorname{sh}\left(\sqrt{\frac{\pi m}{F_{0_{2}}}} X\right) \cos \left(\sqrt{\frac{\pi m}{F_{0_{2}}}} X\right)-\operatorname{sh} \sqrt{\frac{\pi m}{F_{0_{2}}}} \cos \sqrt{\frac{\pi m}{F_{0_{2}}}} \operatorname{ch}\left(\sqrt{\frac{\pi m}{F_{0_{2}}}} X\right) \sin \left(\sqrt{\frac{\pi m}{F_{0_{2}}}} X\right), \\
\text { where } X=\frac{r}{R}-\text { dimensionless coordinate; } \\
F_{O}=\frac{a \tau}{R^{2}} \text { - a criterion for the homochronicity of the field of }
\end{gathered}
$$
the heat transfer potential (the Fourier criterion);

$$
F o_{i}=\frac{\alpha \tau_{i}}{R^{2}} \quad(\mathrm{i}=1,2)
$$

Using the space-time analogy allows us to change the formulation of the problem under consideration and to bring it in a somewhat different form [13-15].

Let the spherical object move along the abrasive surface by rolling with sliding. Let it also be exposed to heat during slip periods (friction against abrasive). And let the abrasive surface have a shape that allows one to distribute "slip spots" evenly over the entire ball surface.

It can be assumed that the model described will allow one to reduce the energy intensity of the process by the discrete supply of heat, if the abrasive is distributed discretely over the contact surface in the most probable zones of appearance of "slip spots". Assuming that technically the organization of such process is possible, it is necessary to solve the problem of the relationship between the parameters of the process and the geometry of the application of the abrasive. In the new formulation of the problem, the full utilization of the processed raw materials (a reduction in the amount of waste) and the preservation of its nutritional value (the absence of an unauthorized rise in the temperature of the processed object) will be the determining indicators of the quality of food processing.

The first question seems to depend essentially on the design of the working bodies and technological regimes, and can only be solved by experience. But to solve the second (observing a certain temperature regime) question, we can use the analytical and numerical estimates that have been performed, changing the boundary conditions of the problem. 
The described physical model can be formalized as the corresponding thermal problem of heat transfer in a rotating ball.

Unlike other models in the case under consideration, the statement of the problem can be written in the following form:

$$
\begin{aligned}
& \frac{\partial t}{\partial \tau}=a\left(\frac{\partial^{2} t}{\partial r^{2}}+\frac{2}{r} \frac{\partial t}{\partial r}\right)-\frac{W}{R} t \\
& \left(\tau>0, \quad 0<r<R, \quad W=\frac{2 \pi R}{n T}\right) \\
& t(r, 0)=t_{0}=\text { const } \quad\left(t_{0}=t_{1}=t_{\text {min }}\right) \\
& t(R, \tau)=\left\{\begin{array}{lll}
t_{1}=t_{\text {min }} & \text { для } & 0<\tau<\tau_{1}, \\
t_{2}=t_{\text {max }} & \text { для } & \tau_{1}<\tau<\tau_{2}=T ;
\end{array}\right. \\
& \frac{\partial t(0, \tau)}{\partial r}=0 ; \quad t(0, \tau)<\infty
\end{aligned}
$$

When solving the problem, one can use the coordinate transformation method, the main idea of which is to find the transformation of the function $t(r, \tau)=u(x, t)$ to a function $w(x, t)$ such that the partial differential equation for the function $w(x, t)$ will be simpler than the original equation.

Unfortunately, there are no universal rules for choosing the appropriate transformation. Under these conditions, one has to use intuitive ideas about the behavior of solutions. The temperature $u(x, t)$ at each point of the object varies due to two factors:

- diffusion of heat along the object (term $\alpha^{2} u_{x x}$ ); $\beta u$ )

- heat transfer through the side surface of the object (term -

Let us note that in the absence of diffusion heat transfer along the object (the temperature at each point of the object will decrease to zero according to the exponential law:

$$
u\left(x_{0}, t\right)=u\left(x_{0}, 0\right) e^{-\beta t}
$$

This circumstance allows us to assume that the solution of the problem can be exploited in the form of a product of two factors:

$$
u(x, t)=w(x, t) e^{-\beta t},
$$

where $w(x, t)$ - temperature distribution due to diffusion.

By substituting, differentiating and bringing the obtained value (13) to (10), let us obtain:

$$
w_{t}=\alpha^{2} w_{x x}
$$

Performing the substitution of expression (13) into the initial and boundary conditions, let us obtain:

$$
w(x, 0)=\varphi(x)
$$

and :

$$
\left\{\begin{array}{l}
w(0, t)=0 \\
w(1, t)=0
\end{array}\right.
$$

The solution of partial differential equations (14) with initial conditions (15) and boundary conditions (16) by the method of separation of variables will have the form:

$$
w(t, x)=\sum_{1}^{\infty} a e^{-(n \pi \alpha)^{2} t} \sin (n \pi x)
$$

where

$$
a_{n}=2 \int_{0}^{1} \varphi(\xi) \sin (\pi n \xi) d \xi
$$

Since the functions $u(x, t)$ and $w(x, t)$ are connected by the transformation (13), the solution of the boundary-value problem (10) - (12) has the form [16, 17]:

$$
u(t, x)=e^{-\beta t} \sum_{n=1}^{\infty} a_{n} e^{-(n \pi \alpha)^{2} t} \sin (n \pi x)
$$

Using this approach, let us get the following solution:

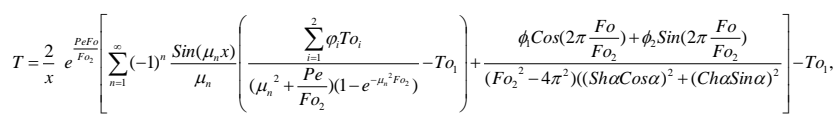


where

$$
\begin{gathered}
\mu_{n}=n \pi ; \\
\varphi_{1}=e^{-\mu_{n}{ }^{2} F o_{2}}\left(e^{\mu_{n}{ }^{2} F o_{1}}-e^{\frac{P e F o_{1}}{F o_{2}}}\right) \\
\varphi_{2}=e^{F o_{2}-P e \frac{F O_{1}}{F O_{2}}}-e^{-\mu_{n}{ }^{2}\left(F O_{2}-F O_{1}\right)}
\end{gathered}
$$$$
\phi_{1}=\gamma\left(M \mathrm{FO}_{2}+2 \pi \mathrm{V}\right)-\left(2 \pi \mathrm{M}-\mathrm{NFO}_{2}\right) \operatorname{Sin}\left(2 \pi \frac{F o_{1}}{\mathrm{Fo}_{2}}\right) ;
$$$$
\phi_{2}=\left(M \mathrm{FO}_{2}+2 \pi N\right) \operatorname{Sin}\left(2 \pi \frac{F O_{1}}{\mathrm{FO}_{2}}\right)-\gamma\left(2 \pi M-N \mathrm{NO}_{2}\right)
$$

$M=\operatorname{ch} \alpha \operatorname{Sin} \alpha \operatorname{ch} \alpha x \sin \alpha x+\operatorname{sh} \alpha \operatorname{Cos} \alpha \operatorname{sh} \alpha x \operatorname{Cos} \alpha x$

\section{$N=\operatorname{ch} \alpha \operatorname{Sin} \alpha \operatorname{sh} \alpha x \cos \alpha x-\operatorname{sh} \alpha \cos \alpha \operatorname{ch} \alpha x \sin \alpha x$} Legend:

$$
\begin{aligned}
& T=T(x, F o)=\frac{t(r, \tau)-t_{1}}{t_{2}-t_{1}} ; \quad(0<r<1) \\
& x=\frac{r}{R} ; \quad F o=\frac{a \tau}{R^{2}} ; \quad F o_{i}=\frac{a \tau_{i}}{R^{2}},(i=1,2) \\
& P e=\frac{W \tau_{2}}{R}=\frac{W T}{R} \\
& \alpha=\sqrt{\frac{\pi}{a \tau_{2}}} R=\sqrt{\frac{R}{F o_{2}}} \\
& \frac{W R}{a}=\frac{P e}{F O_{2}} ; \quad \frac{W \tau_{1}}{R}=\frac{P e F O_{1}}{F O_{2}} \\
& T o i=\frac{t_{i}}{t_{2}-t_{1}} \quad(i=1,2)
\end{aligned}
$$

\section{CONCLUSIONS}

The obtained solutions of the formulated problem for the chosen physical model make it possible to predict the temperature distribution along the depth of the processed object. If some kinds of pro-cessed food raw materials, such as coffee grains, are allowed to influence significant temperatures for a long time, so others (potato tubers, cereals) do not withstand product overheating without compromising quality. Thus, the obtained quantitative estimates of the parameters varied during the processing of raw materials allow solving practical problems for reducing losses, having an effect on the quality of the food product.

The solutions obtained can also be used to solve the inverse problem: to determine the time, not exceeding the critical one, at which an unauthorized excess of temperature is reached. This will enable one to minimize energy consumption providing the most favorable mode of abrasive processing.

\section{References}

[1] D.R. Hartree, Mem. Manchr. Lit. Phil, Soc. pp 80, 85, 1935.

[2] Ногеnstein, Quarterly Journal of Mathematics. App1. Math. 3, pp 183187, 1945.

[3] R. Clemmow, Munford.Phil. Poy. Soc., A 245, pp 189-211, 1952.

[4] Kaye, J. Math. Pbys. 34, pp 119-125, 1955.

[5] G.V. Alexeev, V.N. Krasilnikov, M.S. Kireeva, E.V. Egoshina. "Use of flaxseeds in the flour confectionery", International Food Research Journal,. vol. 3, pp. 1156-1162, 2015.

[6] G.V. Alekseev, A.A. Khripov, "Method of rapid remote control of casein concentration in dairy products in unopened packages", Journal of Food Process Engineering, vol. 1, pp. 11-18, 2015.

[7] G.V.Alexeev, V.N. Krasilnikov, M.S. Kireeva, E.V. Egoshina "Use of flaxseeds in the flour confectionery", International Food Research Journal, vol. 3, pp. 1156-1162, 2015

[8] A.V. Attetkov, I.K. Volkov, "Analytical methods for studying the thermal state of a region with a moving boundary under conditions of nonstationary heat exchange with an external environment, Engineering and Physics Journal, vol. 1 pp. 125-130, 2000.

[9] E. Verboloz, B. Voronenko, A. Pastukhov, G. Alekseev, V. Pelenko, S. Polyakov, "Mathematical modelling of the separation of emulsions through membranes", International Journal for Engineering Modeling, vol. 3-4, pp. 131-135, 2014

[10] B.A. Voronenko, V.V. Klyuchkin, "Analytical study of the temperature field of a layer of oil seeds under controlled temperature effects", MZHP, vol. 3-4, pp. 1-4, 1997.

[11] G.Karslou, D. Eger, "Thermal conductivity of solids", Moscow: Nauka, 1964, p. 488.

[12] A.V. Lykov, "Theory of heat conduction", Moscow: Vysshaya shkola, 1967, p.600.

[13] V.S. Martynenko, "Operational calculus", Moscow: Vyshcha shk., 1990, p. 359.

[14] EH. M. Kartashov, "New integral representations of analytic solutions of boundary value problems of nonstationary transport in domains with moving boundaries", Engineering and Physics Journal, vol. 5, pp. 825836, 1999

[15] EH. M. Kartashov, "Analytical methods in the theory of the thermal conductivity of solids", Moscow: Vysshaya shkola, 2001, p. 550.

[16] EH. M. Kartashov, Analytical methods for solving boundary value problems of nonstationary heat conductivity in regions with moving boundaries (Review)", Engineering and Physics Journal, vol. 2, pp. 171195, 2001. 
[17] EH.M. Kartashov, V.A. Kudinov, "Analytical theory of heat conductivity and applied thermoelasticity", Moscow: URSS, 2012, p.
653. 